\title{
umd-verification: Automation of Software Validation for the EGI federated e-Infrastructure
}

\author{
Pablo Orviz Fernández · João Pina · Álvaro López García · Isabel \\ Campos Plasencia • Mário David · Jorge Gomes
}

This is the authors pre-print version of this work. The final publication is available at http://dx.doi.org/10.1007/ s10723-018-9454-2

\begin{abstract}
Supporting e-Science in the EGI eInfrastructure requires extensive and reliable software, for advanced computing use, deployed across over approximately 300 European and worldwide data centers. The Unified Middleware Distribution (UMD) and Cloud Middleware Distribution (CMD) are the channels to deliver the software for the EGI e-Infrastructure consumption. The software is compiled, validated and distributed following the Software Provisioning Process (SWPP), where the Quality Criteria (QC) definition sets the minimum quality requirements for EGI acceptance. The growing number of software components currently existing within UMD and CMD distributions hinders the application of the traditional, manual-based validation mechanisms, thus driving the adoption of automated solutions. This paper presents umd-verification, an open-source tool that enforces the fulfillment of the $\mathrm{QC}$ requirements in an automated way for the continuous validation of the software products for scientific disposal. The umd-verification tool has been successfully integrated within the SWPP pipeline and is progressively supporting the full validation of the products in the UMD and CMD repositories. While the cost of supporting new products is dependant on the availability of Infrastructure as Code solutions to take over the deployment and high test coverage, the results obtained for the already integrated products
\end{abstract}

Pablo Orviz Fernández - Álvaro López García - Isabel Campos Plasencia

Instituto de Física de Cantabria (CSIC), Santander, Spain

E-mail: orviz@ifca.unican.es

João Pina - Mário David - Jorge Gomes

Laboratório de Instrumentação e Física Experimental de

Partículas (LIP), Lisboa, Portugal are promising, as the time invested in the validation of products has been drastically reduced. Furthermore, automation adoption has brought along benefits for the reliability of the process, such as the removal of human-associated errors or the risk of regression of previously tested functionalities.

Keywords Automation - Software Verification and Validation · Software Quality Assurance - Software Quality Control · Software Testing · Continuous Integration

\section{Introduction}

EGI 1 federates computing and data resources, mainly hosted in Europe, to satisfy common and specific research requirements gathered from multidisciplinary scientific communities. EGI operates as an eInfrastructure 22 that exploits complex data-intensive Grid and Cloud computing services 3,4, through the Unified Middleware Distribution (UMD $\sqrt{1}$ and the Cloud Middleware Distribution (CMD) 2 至 official releases, respectively.

The UMD and CMD distributions provide repositories to distribute software in the form of Linux packages that are provisioned by external technology providers (TPs). The environments in which the software has been developed are not under the control nor monitored by EGI, thus there is no guarantee that the software is reliable enough for the production infrastructures. Therefore, EGI invests on a validation effort for the

\footnotetext{
1 http://repository.egi.eu/sw/production/umd/

2 http://repository.egi.eu/sw/production/cmd-os/

3 http://repository.egi.eu/sw/production/cmd-one/
} 


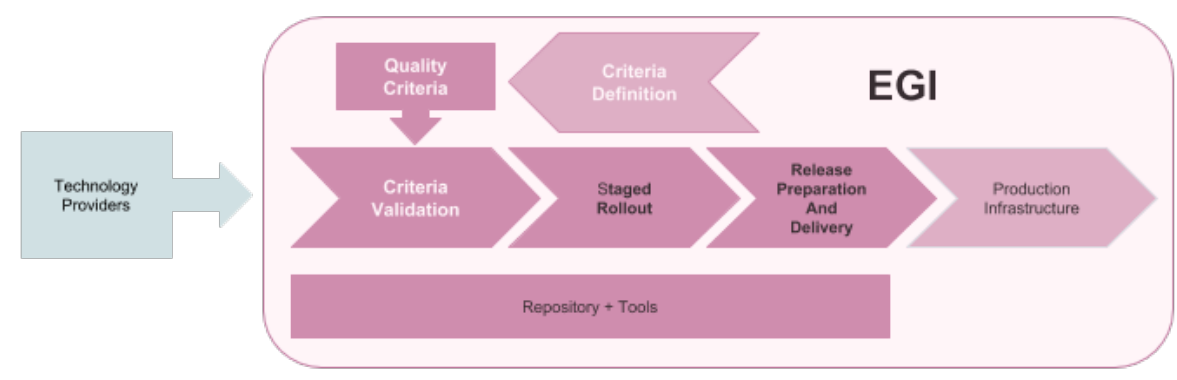

Fig. 1 EGI Software Provisioning Process (SWPP).

incoming software products to lessen the odds of disruption. The Software Provisioning Process (SWPP) [5], schematized in Figure 1, guides the EGI software delivery through UMD and CMD distributions, encompassing the i) validation of the conformance criteria, scope of the present paper, the ii) staged rollout phase, which takes over the deployment and user-level testing on production facilities, and, finally, the iii) release to production, resulting in the software release preparation and delivery.

During the validation of the conformance criteria phase, every piece of software is deployed and tested to detect any malfunction or deviation from the design specification. The procedure of validation is governed by the Quality Criteria (QC) definition, which enforces the quality requirements that any software released under UMD and CMD distributions must comply. The validation phase appears as the most time-consuming task within the SWPP since a major effort is spent on dealing with the deployment peculiarities of each software component, as well as in ensuring a minimal testing coverage. Consequently, the validation process requires some modernization that optimizes the effort invested, being able to respond accurately to the growing needs of UMD and CMD consumers.

The remainder of this paper outlines the automated solution implemented to speed up the process of conformance criteria validation for UMD and CMD products. Section 2 introduces the difficulties of preserving the traditional validation process, presenting automation as a suitable solution for the EGI QC enforcement. Section 3 contextualizes the $\mathrm{QC}$ validation in the software engineering literature, emphasizing the role of automation in the methodologies reviewed. Section 4 introduces the new tool, umd-verification, that drives the QC validation process in an automated fashion. Finally, Section 5 highlights the proven advancements obtained after applying the umd-verification tool in the EGI QC validation process.

\section{Boosting the validation process}

\subsection{Statement of the problem}

An analysis of the evolution of EGI software product catalogue, outlined in Figure 2, shows a growing trend in the number of products being supported since the first release of the UMD distribution, UMD-1. The underlying reasons behind this growth are mainly the evolving technology demands coming from the scientific communities leveraging the EGI e-Infrastructure. Recently, these user requirements resulted in the release of the CMD distribution -as Cloud computing became a popular technology for research computation-, thus considerably increasing the number of products supported in the EGI production catalogue.

It is important to underline that Figure 2 only shows the total products, not the actual validations being performed. The number of validations averagely increases in a factor of 2, depending on the number of operating system (OS) distributions supported within each UMD major release. Table 1 shows the specific OSes supported throughout the UMD and CMD major releases, which in some cases raised up to 3 different OS distributions.

Addressing the growing needs with the former validation process resulted in delays within the SWPP chain, leading to extreme situations where a product release was disregarded and superseded by a subsequent release while queued at this stage. According to [5], the validation of the conformance criteria phase was driven by a team of 15-20 testers, each taking over the product validation process based on their expertise. The process was fully manual, with a typical estimated time completion of 1 or 2 working days for each software validation. Thereby, the traditional approach of QC validation is only sustainable as long as the manpower:number of products ratio remains balanced, which is likely to become unsustainable over time, based on the trend discussed above. 


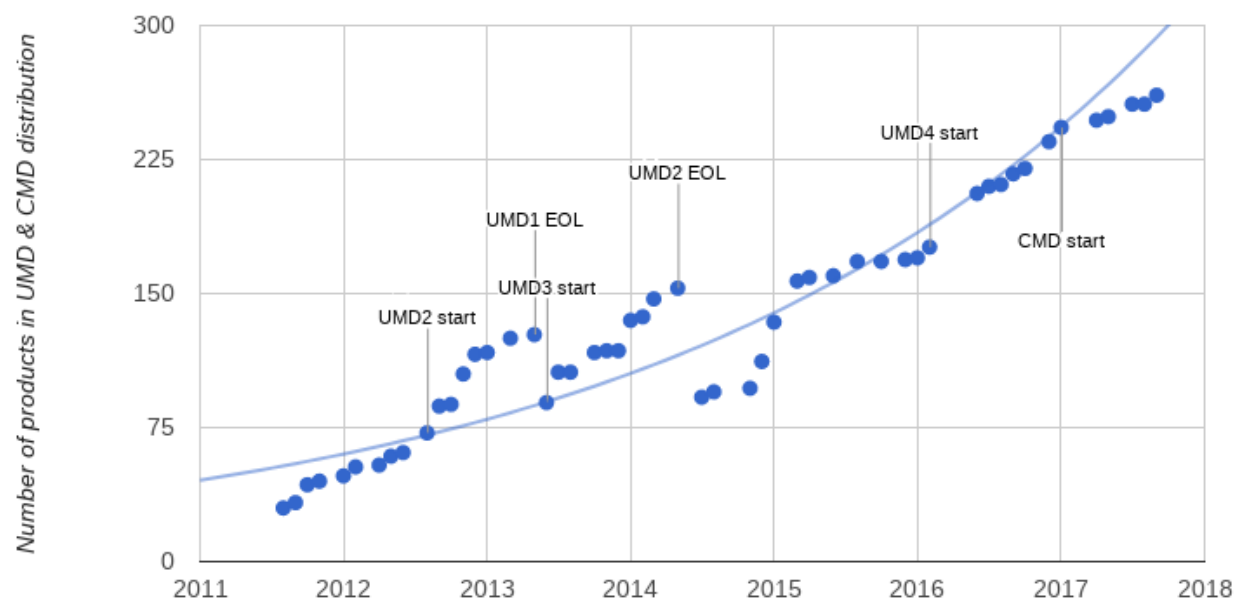

Fig. 2 Trend graph showing the number of products supported in the EGI production repositories (UMD and CMD). The incremental trend is interrupted by the end-of-life (EOL) cycles, which are rapidly recovered as a result of the parallel start of the subsequent major release version. At this point in time, the incoming UMD major release progressively adopts, following the validation process, the products previously existing.

\begin{tabular}{|c|c|c|}
\hline Distribution & Major release & OSes \\
\hline \multirow{6}{*}{ UMD } & $\overline{\text { UMD-1 }}$ & Scientific Linux 5 \\
\hline & & Scientific Linux 5 \\
\hline & $U M D-2$ & Debian Squeeze \\
\hline & UMD-3 & $\begin{array}{c}\text { Scientific Linux } 6 \\
\text { Scientific Linux } 5^{*} \\
\text { Debian Squeeze* }\end{array}$ \\
\hline & & CentOS7 \\
\hline & UMD-4 & Scientific Linux 6 \\
\hline \multirow{4}{*}{ CMD } & \multirow{3}{*}{ CMD-OS } & CentOS7 \\
\hline & & Ubuntu 16.04 \\
\hline & & Ubuntu $14.04^{*}$ \\
\hline & CMD-ONE & CentOS7 \\
\hline
\end{tabular}

Table 1 Operating systems (OSes) supported throughout UMD and CMD distributions lifetime. The support for the OSes marked with an '*', were dropped during the associated release.

\subsection{Embracing automation}

The adoption of automation seems to be an obvious choice to address the delays within the validation phase. This statement rests on the following assumptions.

\section{Manpower}

Taking into consideration the above-mentioned fact of requiring 2 working days for each product:OS validation, in the likely event of having 20 queued products supported in 2 different OSes, approximately 80 working days would be needed to complete their validation. Distributing the load among the 15-20 testers, the process would take roughly a full-time week of work for all the members in the validation team.

While manual validation strongly relies on manpower, an automated approach would only require effort when supporting new products, as the maintenance costs are not highly demanding. Following the current trend, the manual process will soon not scale, requiring more and more testers to satisfy the incoming rate of products.

\section{Expert dependence}

The good progress of a manual software validation is driven by seasoned teams, usually system administrators from resource centers taking part in the EGI eInfrastructure that are highly familiarized with the evaluated product. For this type of validation, technically skilled experts are required to work around unpredictable issues not addressed in the documentation provided by the TPs.

The programmatic implementation of a product validation would only require from expert knowledge the first time it is set up. Once in place, the process could be taken over by non-expert testers since most of the complexity is hidden. This represents a much lighter dependence on skilled testers, being better positioned towards risk of knowledge loss.

Reproducibility and repeatability: fighting the human factor

In the context of mechanical or repetitive processes, the likelihood of human error is substantially higher than when the same process is performed in an automated environment. Whilst automated processes are predictable, humans are not able to work with the same level of consistency.

The deterministic nature of computational solutions makes easier to achieve a high level of repeatability 
in the results obtained when applied to the same input data. Conversely, the same task performed manually could lead to unexpected outcomes as the procedure may not be strictly fulfilled in consecutive iterations. Moreover, the programmatic implementation of a clearly defined iterative procedure, such as EGI's QC, makes the solution reproducible. Therefore, subsequent executions shall obtain the same results as long as the validation process is taken over under the same conditions, regardless of the tester.

\section{Time efficiency}

Automation streamlines the time required to complete a task. Time efficiency is usually associated with automation since it allows to meet strict deadlines or even increase the number of tests that could be performed in the same time slot, resulting in higher test coverages.

\subsection{Automation assessment of the EGI Quality Criteria requirements}

Early introduced, the Quality Criteria (QC) document drives the validation of software products within the SWPP workflow. It defines the quality requirements that a given product has to fulfill in order to be considered ready for the subsequent staged rollout phase. The document is continuously evolving and it is currently on the 7 th release [6].

Table 2 lists the quality requirements, their associated criticality and the possibilities of automation. Requirements cover the minimum criteria for EGI acceptance, and are grouped in seven broad categories: i) documentation, ii) installation, covering the full deployment of the product, iii) security, iv) information model, which validates the outbound data published by the information service, v) operations, which groups probes related to EGI e-Infrastructure, vi) support channels and vii) other specific criteria, useful to extend the functionality and integration testing coverage.

As depicted in the table, the only requirements that need human interaction are the ones related to the analysis of the documentation (QC_DOC_x ): one could address programmatically the existence of the required documentation but not the suitability of its content. Nevertheless, the QC_DOC_x requirements seldom involve major changes -only when products are included for the first time-, commonly appearing as minimal improvements when it comes to software updates.

Once the requirements suitable for automation are identified and defined, the process to tackle them has to be implemented. From the requirement list, deployment and testing related tasks are the most complex and as such will be thoroughly covered in the next sections.

\section{Related work}

Free and open-source software operating systems, such as Linux distributions, rely on packages to distribute the software. Packages are archives containing the binaries, configuration files and dependency information, accessible through online repositories. Software packages can be found in different formats attached to a specific Linux distribution, although there are recent solutions that containeirise software applications, bundling their dependencies, to make them installable across all major Linux distributions [7,8, 9. Most quality-aware distributions have quality control policies for package creation [10] and dependency resolution [11. Likewise Linux operating systems, the software distributed through UMD and CMD releases are in the form of packages, which also are passed through a quality control process. As the latter are lighter distributions, they can afford to go a step further in the software validation, imposing deployment and testing requirements.

Software validation is the process that checks that the software satisfies its intended use, in conformance with the requirements coming from the end users. Tightly related and complemented by the software verification process, they together address "all software life cycle processes including acquisition, supply, development, operation and maintenance", as defined in the IEEE Standard for Software Verification and Validation (V\&V) 12. V\&V are commonplace concepts in software engineering literature, but these terms are often used interchangeably in practice 13 . Indeed, both processes serve different purposes since verification is linked to the early stages of the software development life cycle, focusing on building the software correctly, while validation is commonly placed at the end of the development process, providing "evidence that the software and its associated products satisfy system requirements allocated to software at the end of each life cycle, solve the right problem, and satisfy intended use and user needs". The $\mathrm{V} \& \mathrm{~V}$ distinction is consistent with major systems engineering processes for software development, such as the Capability Maturity Model Integrated [14,15, 16, organized in maturity levels, where software $\mathrm{V} \& \mathrm{~V}$ practices are addressed at the higher levels of the process [17.

A practical way to put $\mathrm{V} \& \mathrm{~V}$ into action is referring to the type of testing associated to each process. Software verification implies the static analysis of the source code, requirements and design documents for defect detection via inspections, walkthroughs and reviews [18. Conversely, software validation requires the software to 


\begin{tabular}{|c|c|c|c|c|}
\hline Category & ID & Check & Critical & Automated \\
\hline \multirow{5}{*}{ Documentation } & $\overline{\text { QC_DOC_1 }}$ & Release notes provisioning & $\overline{\bar{V}}$ & $\overline{\bar{x}}$ \\
\hline & QC_DOC_2 & User documentation & $\checkmark$ & $x$ \\
\hline & QC_DOC_3 & API documentation & $x$ & $x$ \\
\hline & QC_DOC_4 & Admin documentation & $\checkmark$ & $x$ \\
\hline & QC_DOC_5 & Software license & $\checkmark$ & $\checkmark$ \\
\hline \multirow{2}{*}{ Installation } & QC_DIST_1 & Binary distribution (RPM, DEB) & $\checkmark$ & $\checkmark$ \\
\hline & QC_UPGRADE_1 & Upgrade previous working version & $x$ & $\checkmark$ \\
\hline \multirow{6}{*}{ Security } & QC_SEC_1 & X.509 certificate support & $\checkmark$ & $\checkmark$ \\
\hline & QC_SEC_2 & SHA-2 certificate support & $\checkmark$ & $\checkmark$ \\
\hline & QC_SEC_3 & RFC proxy support & $x$ & $\checkmark$ \\
\hline & QC_SEC_4 & ARGUS auth integration & $x$ & $\checkmark$ \\
\hline & QC_SEC_5 & World writable files & $\checkmark$ & $\checkmark$ \\
\hline & QC_SEC_6 & Passwords in world readable files & $\checkmark$ & $\checkmark$ \\
\hline \multirow{3}{*}{ Information Model } & QC_INFO_1 & GLUE schema 1.3 support & $x$ & $\checkmark$ \\
\hline & QC_INFO_2 & GLUE schema 2.0 support & $\checkmark$ & $\checkmark$ \\
\hline & QC_INFO_3 & Middleware version & $x$ & $\checkmark$ \\
\hline \multirow{2}{*}{ Operations } & QC_MON_1 & Service probes & $x$ & $\checkmark$ \\
\hline & QC_ACC_1 & Accounting records & $\checkmark$ & $\checkmark$ \\
\hline Support & QC_SUPPORT_1 & Bug tracking system & $\checkmark$ & $\checkmark$ \\
\hline \multirow{2}{*}{ Specific QC } & QC_FUNC_1 & Basic functionality test & $\checkmark$ & $\checkmark$ \\
\hline & QC_FUNC_2 & New feature/bug fixes test & $x$ & $\checkmark$ \\
\hline
\end{tabular}

Table 2 Quality Criteria (QC) requirements.

be in operation mode to be tested, so it is identified with the dynamic behaviour of the source code. There are different test-case design methodologies to tackle the dynamic analysis of a software component but all fall in the category of so-called black-box testing. In this type of testing the test-cases are data or input/output driven, as the internal structure of the software is not of interest at this stage. In this regard, Myers et al. [19] group under the term higher-order testing the type of black-box testing methods -function, system, installation, integration, acceptance- that aim to detect defects, from the user's perspective, by categorizing the test cases in which the software shall be exposed. The outcome is a quality criteria that guide the software validation.

The ultimate goal of software validation is to increase the reliability of the systems being delivered to the users. Nevertheless, in software validation, the economics of testing shall be carefully considered. On the one hand, inadequate investment may imply solving defects at later stages. Quoting from Perry's book 20, "it is at least 10 times as costly to correct an error after coding as before, and 100 times as costly to correct a production error". On the other hand, a generous effort may lead to increased project costs [21], not estimated in the project design, and delays in the release dates 22. Therefore, measuring the cost-effectiveness of the testing process does not only imply stopping at the optimum point where the cost of testing does not exceed the value obtained from the defects uncovered, but also focusing on the valuable features first within the appropriate testing phase in the life cycle [23].
Test automation is gaining momentum as a way to decrease the costs and time associated to software testing tasks. Process efficiency gets improved as automation optimizes the execution time of testing, maximizing the test coverage as more testing could be performed in less time [24]. The augmentation of the test coverage strengthens the quality and reliability of the end product, reducing the number of defects present. Automation also increases the overall effectiveness, avoiding the risk of human errors and achieving repeatability. This is particularly useful to reduce the regression risk by finding defects in the modified, but previously working, functionalities of the system 25.

However, test automation does not always supersede manual testing. According to a number of studies 26, 27, 28, not all the testing tasks can be easily automated, such as those requiring extensive knowledge in a specific domain, or they require a costly maintenance. In some cases, manual testing can complement automation since, based on its unstructured nature, it could potentially expose unexpected defects not considered in the previous stages within the software life cycle.

\section{4 umd-verification: an automated tool for the software validation process}

In order to automatize the software validation process within EGI, the essential component would be a general purpose tool to manage the QC execution for each product validation. This tool would execute the appropri- 


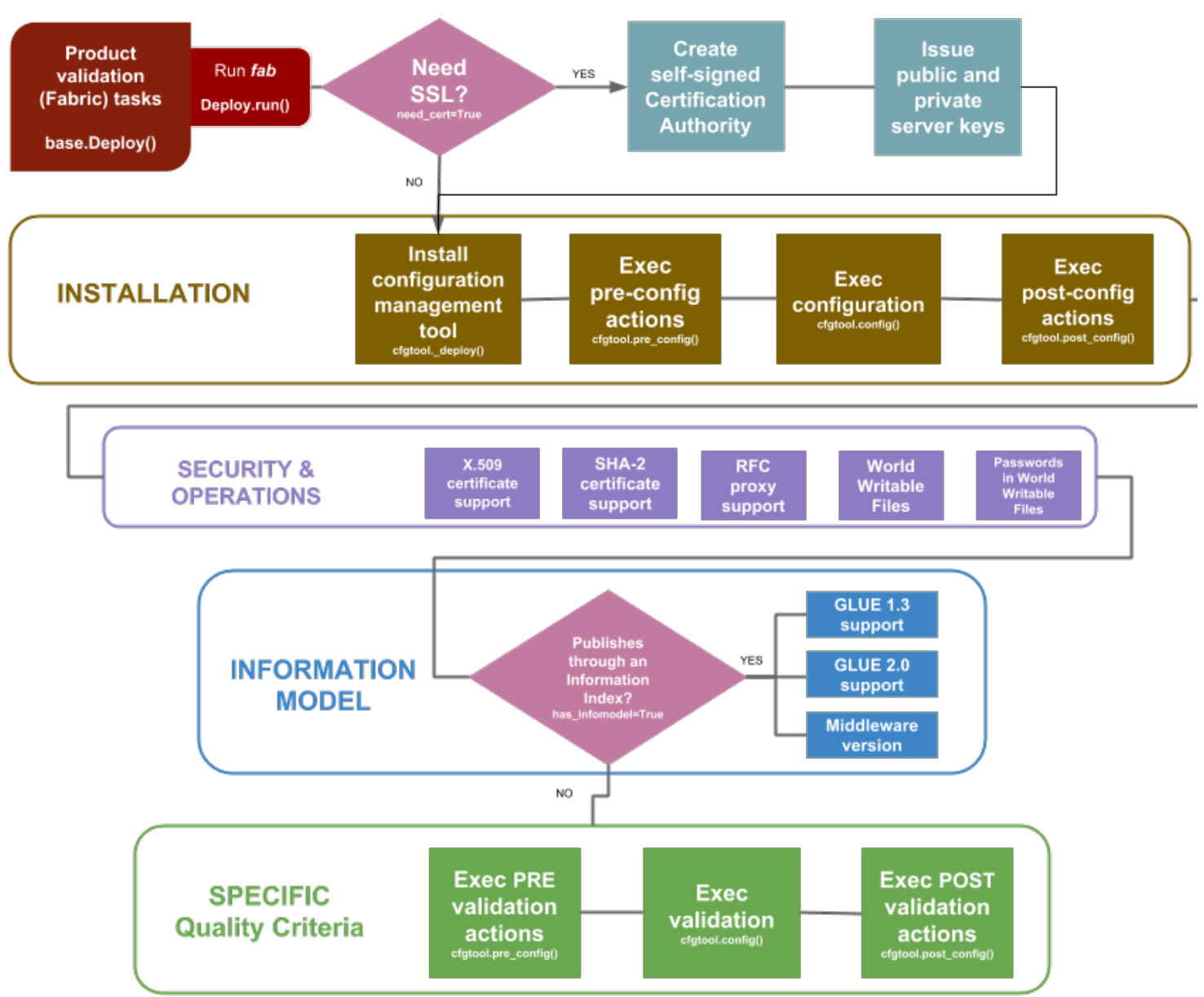

Fig. 3 Product validation worflow in umd-verification.

ate tasks for each requirement analysis and, eventually, evaluate the obtained output values to judge whether the given requirement has been fulfilled, allowing the process to stop depending on its criticality.

\subsection{Design considerations}

\section{Infrastructure as Code deployments}

With the advent of Infrastructure as code ( $\mathrm{IaC}$ ) tools, the automated maintenance and provision of services in an infrastructure is powered through a series of definition files, which enforce the desired configuration of such services. Applying the IaC model to drive the deployment part of the SWPP process would allow to have reliable, repeatable and reusable configurations that supersede the traditional, less-efficient, manual guided deployments. The solution to be implemented shall ensure the usage of common, well-known IaC tools such as Ansible 29] or Puppet 30].

\section{Functional and integration testing}

The QC definition enforces the fulfillment of functionality testing, which covers the newly added features and bugfixes. In this regard, the tool that orchestrates the QC validation shall be flexible enough to execute external scripts, wait for their completion and approve the exit status.

Integration testing is needed whenever the product in validation interferes with additional services while in operation. This type of testing requires more complex deployments, as the related services must be in place in advance.

\section{Dynamic provision of input parameters}

Input parameters are needed in order to set up the diverse environments in which the currently existing products are verified. The application managing the process needs to be fed with several types of input parameter provision, such as run-time arguments, instantiation-time parameters and configuration files. 


\section{Inclusion of new products}

Based on the incremental trend of product adoption in the EGI software distributions, the integration of new products into the automated solution proposed shall be an easy task. The system shall provide a way to declare new products in a standard way, relying on an ubiquitous language that requires little or no previous experience from the tester.

\subsection{Implementation of umd-verification tool}

umd-verification tool 31 is the solution proposed for the automated, sequential validation of the requirements defined in the QC document. The tool is written in the Python programming language [32] and uses the Fabric library 33 for a high-level management of the system calls. Fabric-ed applications are organized in tasks and have built-in features such as remote executions and consistent argument passing via the commandline fab tool.

\section{Behind the scenes}

Figure 3 shows the tool's workflow. Every new product validation is represented by an instance of the customized Fabric base task, base.Deploy, which guides the process through four major execution blocks: i) installation and configuration, ii) security and operations, iii) information model, and iv) specific QC. Note that, as already commented in Section 2.3 documentation requirements need of human revision and thus are not being validated by the application.

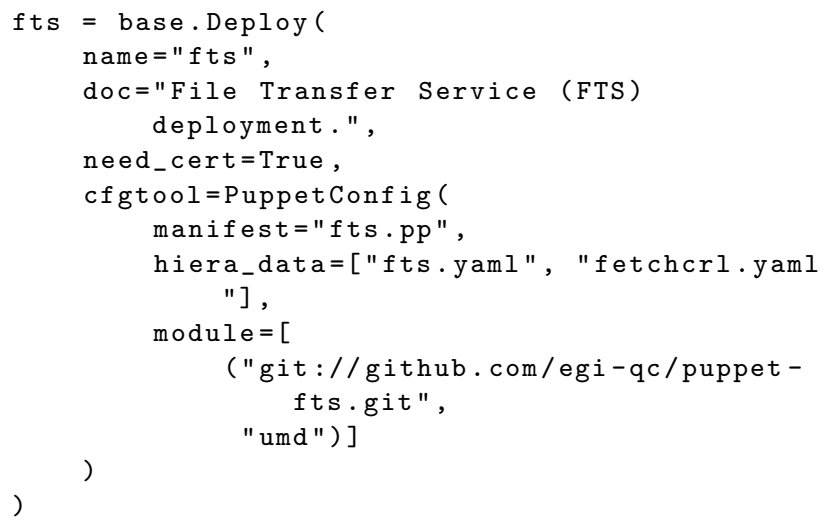

Listing 1 Python code snippet taken from the task validation of $\mathrm{fts}$ product. The class attributes contain static information such as the relevant pointers to enable the product's deployment using Puppet.

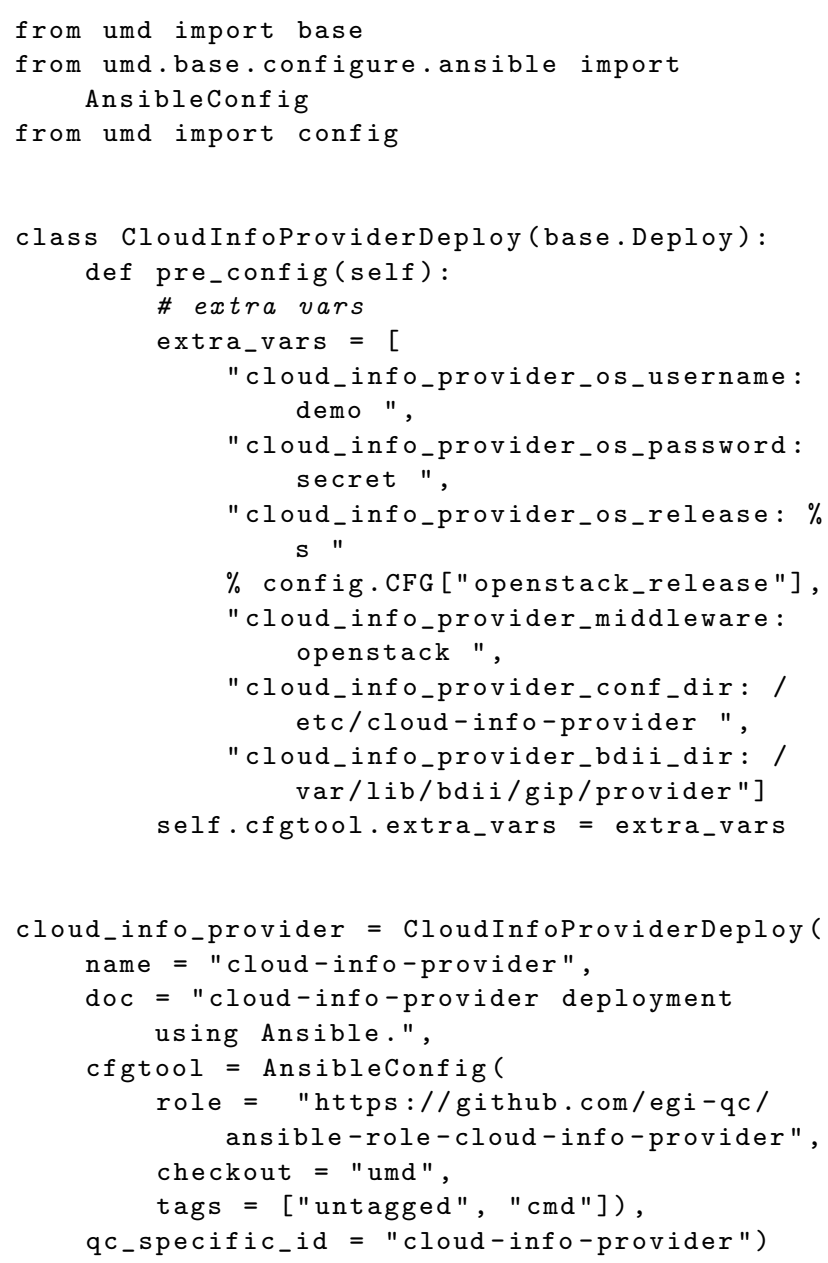

Listing 2 A complete task definition (base.Deploy) for the validation of cloud-info-provider product (Python code). The task relies on an Ansible role for the deployment, which needs a set of input variables that are defined within the pre_config method. The testing part is defined in an external configuration file (see Listing 3), identified by the cloud-info-provider label.

As a result of being inherited from the base class base. Deploy, every product validation need to provide a set of class attributes that uniquely identify the product. The code excerpt from Listing 1 shows a sample implementation of a task validation. One of these class attributes sets the next step in the workflow. A very common requirement for Cloud and Grid services supported in EGI is to guarantee user data protection by securing the connections using X.509 certificates [34. Hence the definition of the need_cert attribute, which when enabled, issues a server certificate from a selfsigned certification authority.

The first block, Installation, addresses the deployment from scratch of the product using an IaC solution. The base.Deploy._deploy() method first installs the $\mathrm{IaC}$ tool and sets the required environment, such as generating parameter files and handling the module in- 
stallation and its dependencies. The deployment is then triggered through the base.Deploy. config() method, with optional pre and post steps that could have previously defined at instantiation time.

The Security and Operations block is comprised of a set of basic security assessments. This phase is specially significant for the secured products since it checks the compliance with X.509 cryptographic standard and SHA-2 signatures 35.

Workload orchestration within EGI e-Infrastructure relies on the resource information published by the providers. The Information Model block ensures the presence of published resource information, in GLUE format 36, validated by the execution of an external tool, glue-validator 37. As not all the supported products in UMD and CMD publish GLUE data, the class attribute has_infomodel signals when this requirement should be checked.

The last block, Specific Quality Criteria, covers the functional and/or integration testing of the product. Here, basic operation and new features and/or bugfixes included in the release are tested. The class attribute qc_specific_id maps to the set of checks, in the form of scripts, that must be executed. In the subsequent product validations, these checks eliminate the regression risk as they are re-executed to ensure that the previous working functionalities are kept.

Listing 2 shows a more advanced usage of a validation task. In this example the base. Deploy .pre_config ( method is overridden to set the values of some parameters that need to be defined before the product's deployment using Ansible. Moreover, the task is completed with a test definition through the qc_specific_id attribute.

\section{Support for new software components}

One of the key design considerations of umd-verification application was to ease the addition of new product validations, while relying on a powerful and ubiquitous language. As described in the section above, the usage of the Python language matched both design requirements with the only caveat of assuming certain degree of experience in Python programming, specially in the case of very customized and complex configurations.

The simplest case would directly inherit from the base class base.Deploy, while more complex scenarios would create a child class, overriding the necessary class methods and attributes as shown in Listing 2. In either case, a new task definition is added by filling in the mandatory attributes, consisting in the name and description, the IaC configuration repre- sented by the cfgtool attribute and the associated test checklist identified by the qc_specific_id attribute. Enabling or disabling the optional attributes need_cert and has_infomodel further define the validation task and, consequently, the workflow to be followed in the Security and Information Model blocks.

Deployment settings vary with respect to the $\mathrm{IaC}$ tool in use, having each a different object class that takes over the deployment based on the parameters passed, and accessible through the base.Deploy.cfgtool attribute. Listings 1 and 2 use different cfgtool objects, representing Puppet and Ansible respectively.

Product testing needs a definition where the checklist of tests are listed in order to be triggered in the task validation. Listing 3 shows an excerpt of the configuration file used for the test definitions. Tests are categorized by the QC requirement - either QC_FUNC_1 or QC_FUNC_2- and defined by the test description, location and arguments.

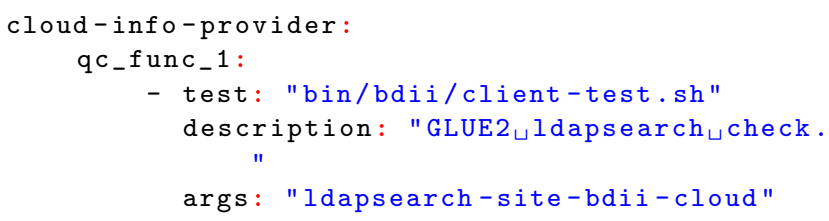

Listing 3 cloud-info-provider YAML test definition.

Based on the above guidelines, new product adoption within the umd-verification tool is not a costly task whenever the IaC modules and tests are already available, either provided by the TPs or individuals that share their work publicly. Both deployment and testing are time-consuming tasks, if performed from scratch, that require a great deal of expertise in the candidate product and, additionally, in the IaC tool being used.

\section{Evidence of the umd-verification adoption}

\subsection{Continuous Integration implementation}

umd-verification is suitable for being integrated in a Continuous Integration (CI) pipeline. The CI system fires up the virtual resource, sets up the application, triggers the execution with the appropriate runtime parameters and, finally, tears down the provisioned resource. All these steps are condensed in a job definition within the Jenkins CI service 38] for each product in the catalogue. Figure 4 shows a sample form in Jenkins CI that, on submission, will trigger the validation process leveraging the umd-verification tool. The runtime parameters passed are commonly the ones showcased in the figure, consisting in the EGI distribution 


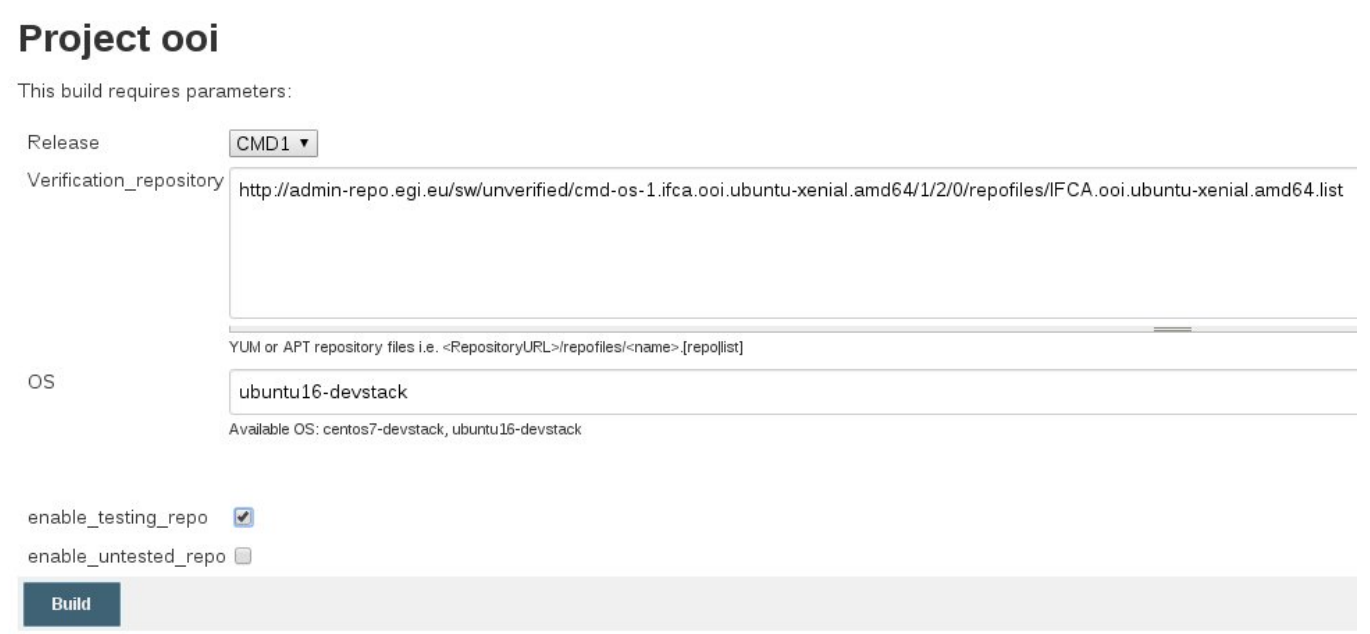

Fig. 4 Product validation form in Jenkins. The input (runtime) parameters are comprised of the software release -from UMD or CMD distributions-, the operating system and the URL of the verification repository that contains the software packages to be validated.

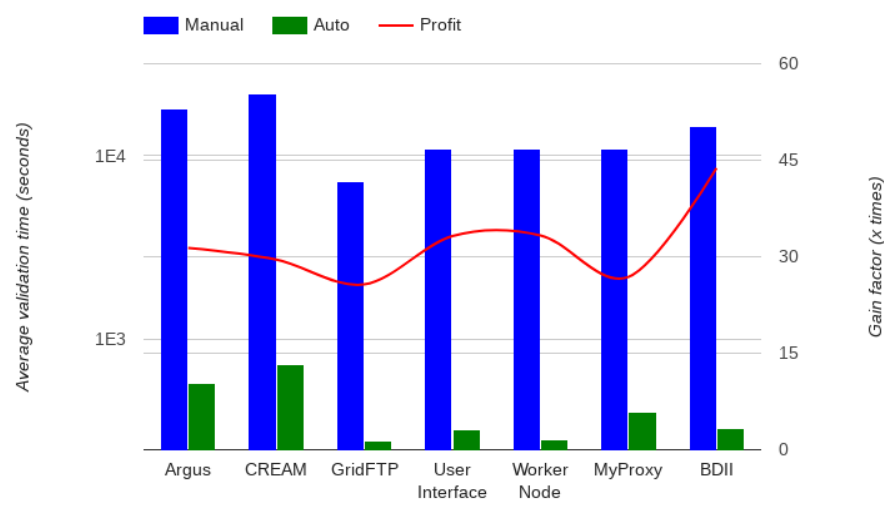

Fig. 5 Automated vs Manual validation process times. Time values on the vertical axis use a logarithmic scale to better showcase the important differences of time completion for both types of validation processes. Manual values are not as accurately estimated as the automated ones. Whilst the latter have been obtained from the CI service, the manual values were extracted from the validation reports uploaded to the EGI Document Database 39.

and Linux OS, and the additional repositories, such as the one containing the candidate version of the software product.

The usage of a CI service to take over the validation of products, notably hides the inner complexity of the validation process -resource provisioning, umd-verification deployment and execution-, allowing a non-expert usage.

\subsection{Time efficiency for the validation process}

The paramount benefit of automating the validation process via the umd-verification application is the time efficiency. Combined with the automated resource provisioning, provided by the CI implementation previously described, this efficiency raises even higher.

As it was mentioned in the statement of the problem in Section 2.1, back in the days of the manual validation process [5], a common completion time was estimated to be 1 or 2 days. With the new approach the validation process takes a few minutes, although this duration is tightly related to the deployment requirements of each software component, as some products need additional services for the testing phase. Therefore, the time required to add support for a new product within the umd-verification tool may be costly whenever there is no availability of $\mathrm{IaC}$ modules. Otherwise, as it was shown in Section 4.2 , the definition of the new product in Fabric is an immediate task.

The data displayed in Figure 5 compares the validation time of both approaches for a set of UMD products, showcasing the profit percentage obtained with the automated process. The results show an average factor of 32 in the time efficiency of the validation process with the adoption of the automation process described throughout this paper.

\subsection{IaC knowledge base}

One of the requirements imposed when supporting a new product validation in the umd-verification application is the usage of an IaC solution for its deployment. Since the adoption of automation, the EGI validation team maintains a public repository [40] with a collection of Ansible and Puppet modules resultant from the validation process. Figure 6 provides an overview of the work being done in this regard and referred as maintained -modules created and supported 


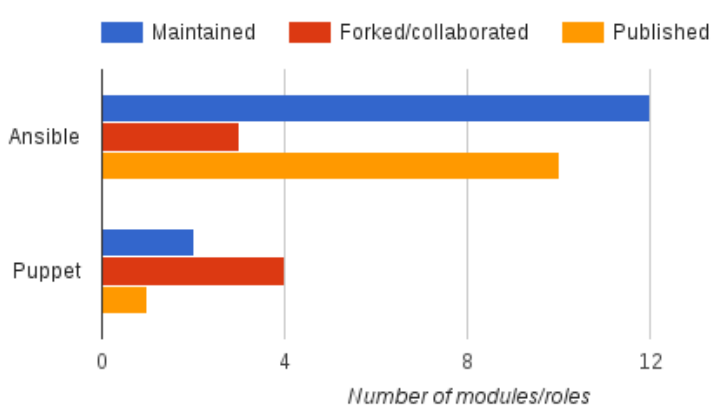

Fig. 6 Ansible roles and Puppet modules being maintained, forked and published in the official repositories by the EGI validation team.

by the EGI validation team-, forked -modules modified and contributed to upstream- and published -modules contributed to the official Ansible [41] and Puppet 42] community repositories-.

As self-documentation code, IaC modules shaped in the validation phase can be then re-used in a reproducible way in future deployments. As a result, within the EGI e-Infrastructure, resource center operators can make use of those modules to deploy the products in the EGI catalogue. This contrasts with the previous procedure, where deployments done in the validation phase could not be easily reproduced: they were locally addressed by the tester, with the only reference of a set of non-structured annotations being included in the verification report.

\subsection{Release Candidate validation}

Contiguous software validations are packed in releases, each defined by a version number that reflects its purpose either as revision, minor or major release. Every release is checked by the EGI validation team before being announced as production-ready, following the $R e$ lease Candidate $(\mathrm{RC})$ procedure.

The early $\mathrm{RC}$ implementation relied on a script file that verified the installation of the EGI product catalogue 43 . The list of products was manually maintained, adding or removing entries in the script as the catalogue evolved. With the advent of the umd-verification tool and the adoption of IaC capabilities, the validation of the RCs is eventually tackled using an Ansible role [44. This new implementation fetches dynamically the whole set of packages of either UMD or CMD repositories, to detect any unresolved dependency that might be introduced by the new packages that take part of the release. Whenever detected, the validation team fixes the dependency issue and re-runs umd-verification tool until all the packages in the repository are properly installed.
The dynamic gathering of packages profits from Linux package management utilities, thus there is no need to maintain a static list of software packages to install for each RC. Furthermore, in the past this list did not contain the complete set of packages but only the ones that refer to the main products in the catalogue. As a result, there was a potential risk of uninstallable packages living at the EGI repositories. As it can be seen in Figure 7, almost 25 revisions -since 2012- were explicitly devoted to solve troubles in package dependencies. With the new implementation -back in July 2017- the number of revision releases meant for dependency resolution dropped to zero.

\section{Conclusions}

Based on the growing demands of adopting new products and supporting existing ones in the UMD and CMD releases, the validation of the conformance criteria has to move forward to an automated process. The suitability of the requirements currently existing in the EGI QC document to be addressed programmatically paved the path to the implementation and further integration of the umd-verification tool within the EGI SWPP. The current set of existing products in UMD and CMD repositories are being progressively integrated in the new automated process, often at the cost of developing the required automated deployment and test cases whenever they are not provided directly by the technology provider or shared within the community. However, for those products already integrated, the evidence of improvement has been demonstrated both in terms of efficiency, as the process validation times are clearly optimized, and effectiveness, by getting rid of the likelihood of human error.

Completion time efficiency is the most apparent benefit of adopting automation, shortening the process in an average factor of approximately 32 when compared with the reported time of traditional manual validations. This implies less human effort than the former approach, now reallocated to integration and maintenance activities, being in a better position to confront unexpected demands of product validations. Growing needs of manpower are no longer the solution to high demands as, once the cost of integrating the product in the umd-verification tool is assumed, it will require little or no human intervention.

The programmatic evaluation of the EGI QC requirements combined with the adoption of IaC solutions achieved repeatability and reproducibility in the process of validating software. In particular, IaC modules make the deployment of the products to be reproduced and shared, contributing to the creation of a knowledge 


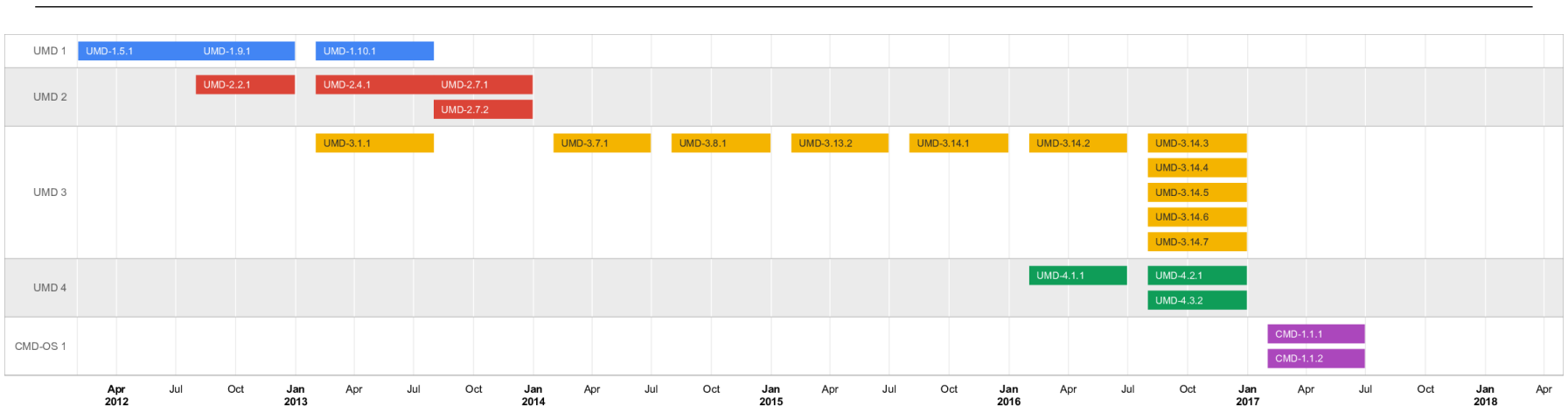

Fig. 7 UMD and CMD revision releases that fixed previous releases with package dependency issues included. As of April 1st 2018, no revision release was needed to solve package unmet dependencies.

base within the community. Just as the IaC modules facilitate the products' deployment to non-experienced users, so does the umd-verification solution with the validation of conformance criteria. Testers not familiarized with a given product can now take over its validation without any expert intervention and, as a result of enabling umd-verification within a CI scenario, even testers with no previous experience with the tool ought to complete the validation process. This accessibility has remarkably reduced the risk of expert dependence, which was tightly associated to the former manual process.

Acknowledgements This work has been partially funded by the EGI-Engage project (Engaging the Research Community towards an Open Science Commons) under grant agreement No. 654182. The authors are especially grateful to EGI.eu's colleagues Enol Fernández, for his contributions to the umd-verification codebase, and Vincenzo Spinoso, for his support in the tool integration within the EGI Software Provisioning Process.

\section{References}

1. EGI.eu. EGI Federation. https://www.egi.eu, 2018. Online; accessed April 1st, 2018.

2. G. et al. Andronico. E-infrastructures for e-science: A global view. Journal of Grid Computing, 9(2):155-184, 2011.

3. Shamsi J. et al. Data-intensive cloud computing: Requirements, expectations, challenges, and solutions. Journal of Grid Computing, 11(2):281-310, 2013.

4. Pérez M.S. Montes J., Sánchez A. Riding out the storm: How to deal with the complexity of grid and cloud management. Journal of Grid Computing, 10(3):349-366, 2012.

5. David M. et al. Validation of grid middleware for the european grid infrastructure. Journal of Grid Computing, 12(3):543-558, 2014.

6. EGI Quality Assurance team. EGI Quality Criteria 7th release. http://egi-qc.github.io/, 2018. Online; accessed April 1st, 2018.

7. Simon Peter. AppImage. https://appimage.org/ 2018. Online; accessed April 1st, 2018.
8. Ubuntu. Ubuntu Snap. https://www.ubuntu.com/ desktop/snappy, 2018. Online; accessed April 1st, 2018.

9. FlatPak. FlatPak. https://flatpak.org/, 2018. Online; accessed April 1st, 2018.

10. Debian. Debian Policy Manual. https://www.debian.org/ doc/debian-policy/ 2018. Online; accessed April 1st, 2018.

11. Debian. Debian Quality Assurance. https://piuparts. debian.org/, 2018. Online; accessed April 1st, 2018.

12. IEEE Computer Society. Ieee standard for system and software verification and validation. IEEE Std 1012-2012 (Revision of IEEE Std 1012-2004), pages 1-223, 2012.

13. Michael J Ryan and Louis S Wheatcraft. On the use of the terms verification and validation. In INCOSE International Symposium, volume 27, 1, pages 1277-1290. Wiley Online Library, 2017.

14. CMMI Product Team. Cmmi for development, version 1.3. Technical Report CMU/SEI-2010-TR-033, Software Engineering Institute, Carnegie Mellon University, Pittsburgh, PA, 2010.

15. CMMI Product Team. Cmmi for services, version 1.3. Technical Report CMU/SEI-2010-TR-034, Software Engineering Institute, Carnegie Mellon University, Pittsburgh, PA, 2010.

16. CMMI Product Team. Cmmi for acquisition, version 1.3. Technical Report CMU/SEI-2010-TR-032, Software Engineering Institute, Carnegie Mellon University, Pittsburgh, PA, 2010.

17. P. Monteiro, R. J. Machado, and R. Kazman. Inception of software validation and verification practices within cmmi level 2. In 2009 Fourth International Conference on Software Engineering Advances, pages 536-541, Sept 2009.

18. Andy German. Software static code analysis lessons learned. Crosstalk, 16(11):19-22, 2003.

19. Tom Badgett Glenford J. Myers and Corey Sandler. The Art of Software Testing. John Wiley \& Sons, Inc., Hoboken, New Jersey, 2012.

20. William E Perry. Effective methods for software testing: Includes complete guidelines, Checklists, and Templates. John Wiley \& Sons, 2007.

21. Edward Kit. Software testing in the real world: improving the process. Addison-wesley, 1995.

22. Chin-Yu Huang and Michael R Lyu. Optimal release time for software systems considering cost, testing-effort, and test efficiency. IEEE transactions on Reliability, 54(4):583591, 2005.

23. James Bullock. Calculating the value of testing from an executive's perspective, software testing is not a capital investment in the physical plant, an acquisition, or 
another readily accepted business expense. a quality assurance manager describes how to present testing as a business-process investment. Software Testing and Quality Engineering, 2:56-63, 2000.

24. Francesca Saglietti and Florin Pinte. Automated unit and integration testing for component-based software systems. In Proceedings of the International Workshop on Security and Dependability for Resource Constrained Embedded Systems, page 5. ACM, 2010.

25. Elfriede Dustin, Jeff Rashka, and John Paul. Automated software testing: introduction, management, and performance. Addison-Wesley Professional, 1999.

26. Dudekula Mohammad Rafi, Katam Reddy Kiran Moses, Kai Petersen, and Mika V Mäntylä. Benefits and limitations of automated software testing: Systematic literature review and practitioner survey. In Proceedings of the 7th International Workshop on Automation of Software Test, pages 36-42. IEEE Press, 2012.

27. Kristian Wiklund, Sigrid Eldh, Daniel Sundmark, and Kristina Lundqvist. Impediments for software test automation: A systematic literature review. Software Testing, Verification and Reliability, 27(8), 2017.

28. Ossi Taipale, Jussi Kasurinen, Katja Karhu, and Kari Smolander. Trade-off between automated and manual software testing. International Journal of System Assurance Engineering and Management, 2(2):114-125, 2011.

29. Ansible. Ansible. https://www.ansible.com/, 2018. Online; accessed April 1st, 2018.

30. Puppet. Puppet. https://puppet.com/, 2018. Online; accessed April 1st, 2018.

31. EGI Software Provisioning team. umd-verification tool. https://github.com/egi-qc/umd-verification 2018. Online; accessed April 1st, 2018.

32. The Python Community. The Python language. https:// www.python.org/, 2018. Online; accessed April 1st, 2018.

33. Jeff Forcier. Fabric - Pythonic remote execution. http:// www.fabfile.org/, 2018. Online; accessed April 1st, 2018.

34. D. Cooper, S. Santesson, S. Farrell, S. Boeyen, R. Housley, and W. Polk. Internet x.509 public key infrastructure certificate and certificate revocation list (crl) profile. RFC 5280, RFC Editor, May 2008. http://www.rfc-editor. org/rfc/rfc5280.txt

35. National Institute of Standards and Technology (NIST). Secure hash standard. Federal Inf. Process. Stds. (NIST FIPS), pages 180-4, 2015.

36. Open Grid Forum. GLUE Specification v. 2. https: //www.ogf.org/documents/GFD.147.pdf, 2018. Online; accessed April 1st, 2018.

37. CERN. GLUE validator guide. http://gridinfo.web. cern.ch/glue/glue-validator-guide, 2018. Online; accessed April 1st, 2018.

38. Jenkins. EGI Jenkins CI. https://jenkins.egi.ifca.es/, 2018. Online; accessed April 1st, 2018.

39. EGI.eu. EGI Document Server. https://documents.egi. eu/, 2018. Online; accessed April 1st, 2018.

40. EGI Software Provisioning team. EGI Quality Criteria in GitHub. https://github.com/egi-qc 2018. Online; accessed April 1st, 2018.

41. EGI Software Provisioning team. EGI Quality Criteria in Ansible Galaxy. https://galaxy.ansible.com/egi-qc/, 2018. Online; accessed April 1st, 2018.

42. EGI Software Provisioning team. EGI Quality Criteria in PuppetForge. https://forge.puppet.com/egiqc/, 2018. Online; accessed April 1st, 2018.

43. Álvaro Simón. EGI Release candidate tester. https: //github.com/alvarosimon/RC_tester, 2018. Online; accessed April 1st, 2018.
44. EGI Software Provisioning team. EGI Release candidate Ansible role. https://github.com/egi-qc/ ansible-release-candidate, 2018. Online; accessed April 1 st, 2018. 